Ann. Biol. anim. Bioch. Biophys., 1979, 19 (4 A), 1007-1010.

\title{
Description d'une technique permettant d'effectuer des prélèvements répétés de gaz dans le rumen
}

\author{
par J. P. JOUANY, J. SENAUD * \\ avec la collaboration technique de J. FLECHET, J. LEFAIVRE \\ Laboratoire de la Digestion des Ruminants, INRA \\ Theix, Saint-Genès-Champanelle, 63110 Beaumont, France. \\ * Laboratoire ossocié au CNRS no 138, Biologie comparée des Protistes \\ Universifé de Clermoni II, Les Cézeaux, B. P. 45, 63170 Aubière, France.
}

Summary. A technique for repeated sampling of rumen gases.

We have described a rumen cannula and a method for sampling rumen gases to be analyzed during digestive or metabolic studies. The procedure is simple and gives samples without contamination by air during 5 hrs after feeding. Frequent gas samples (per minute) can be obtained, thus permitting kinetic studies of rumen gas composition without disturbing the animals.

\section{Introduction.}

La détermination de la composition des gaz produits par le métabolisme des microorganismes du rumen est une opération délicate car la plupart des méthodes de prélèvement des gaz décrites ne sont pas fiables. In vivo deux techniques de prélèvement sont utilisées :

- La première consiste à introduire, sur le flanc gauche de l'animal, un trocart qui débouche au niveau de la partie supérieure du sac dorsal du rumen (Pilgrim, 1948 ; Miltimore ef Mc Arthur, 1962) ; elle ne permet pas d'effectuer un nombre important de prélèvements au cours de la journée sur le même animal car elle peut entraîner la formation d'abcès sous-cutanés. Ainsi il est difficile de réaliser des études sur la cinétique postprandiale de la production des gaz du rumen.

- La seconde nécessite l'emploi d'animaux fistulés ; les gaz sont alors prélevés à l'aide de flacons maintenus sous faible pression et reliés à la canule du rumen d'une manière étanche (Knox, Black et Kleiber, 1969). Par cette technique plus difficile à mettre en œuvre, nous avons foujours obtenu, lors de prélèvements répétés, des échantillons contaminés par de l'air provenant des manipulations nombreuses au niveau de la canule. C'est pourquoi nous proposons une technique de prélèvement à la fois simple et précise qui, en outre, ne perturbe pas les animaux. 


\section{Mode opératoire.}

17 moutons adultes de différentes races, porteurs de fistules permanentes du rumen ont été utilisés. Ils recevaient un régime contenant 40 p. 100 de luzerne déshydraté, 50 p. 100 d'orge, 9 p. 100 de paille de blé et 1 p. 100 de complément minéral et vitaminique. Des échantillons gazeux ont été prélevés à la fois par la méthode décrite (sur 14 moutons) et par ponction dans le sac dorsal du rumen (sur 3 moutons) afin de comparer les deux techniques.

Technique de prélèvement proposée (fig. 1).

Le corps d'une canule du rumen classique (1), maintenu en place sur l'animal par deux rondelles (2) et (3) qui enserrent la fistule, porte un bouchon modifié : un tube traversant ce dernier dans son axe principal (5) débouche dans le rumen. A

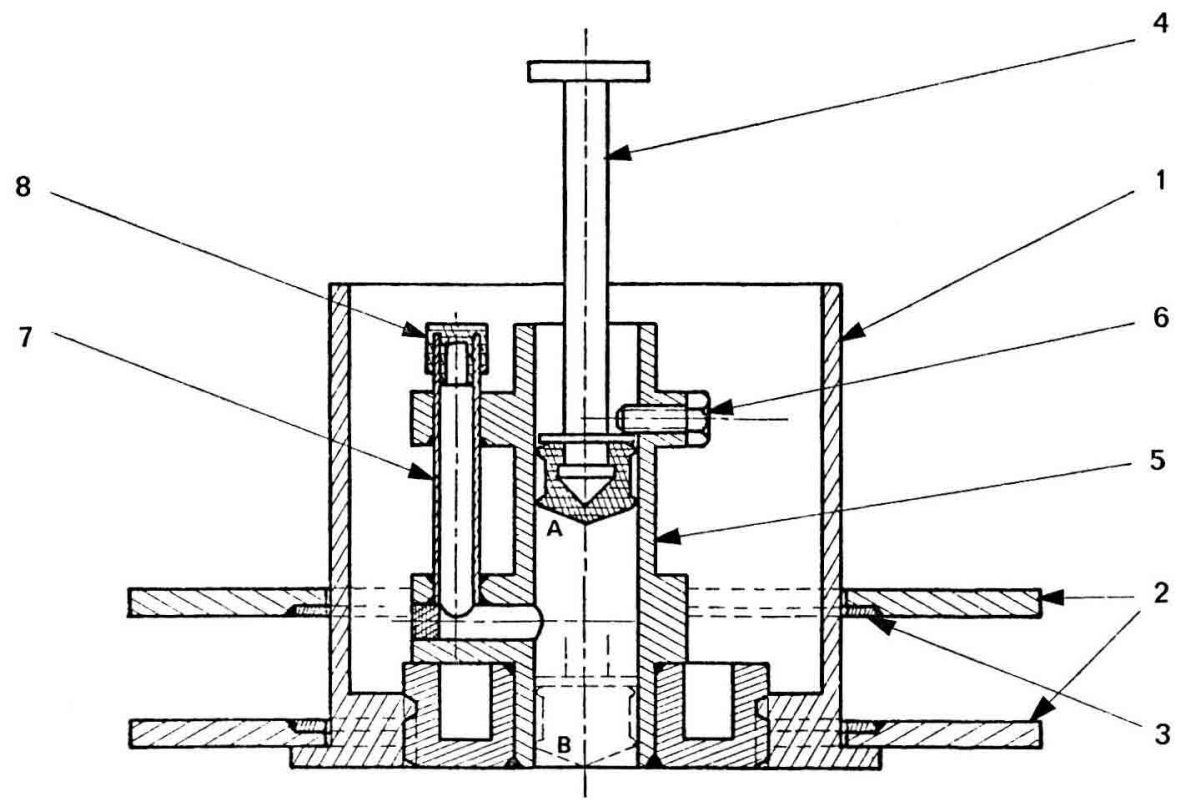

FIG. 1. - Canule du rumen pour prélèvement de gaz. 1) Corps de la canule en nylon. 2) Rondelles d'étanchéité souples en Plastisol. 3) Armature rigide des rondelles d'étanchéité en PCV. 4) Piston muni d'un joint d'étanchéité en caoutchouc à son extrémité. 5) Bouchon de la canule. 6) Vis de butée fin de course du piston. 7) Tube de prélèvement en plexiglass transparent. 8) Capsule en latex.

l'intérieur de ce tube se déplace un piston (4) muni à son extrémité d'un joint d'étanchéité en caoutchouc. Ce conduit (5) communique par sa base avec un tuyau latéral (7) de section plus faible, en plexiglass transparent, obstrué à son orifice extérieur par un bouchon en latex (8). 
Au moment du prélèvement, on appuie sur le corps de la canule de manière à en éloigner le contenu du rumen alors que le piston (4) est en position B. On tire ensuite sur le corps de la canule en amenant le piston en position $A$. Le conduit (7) communique ainsi avec l'intérieur du rumen. Il suffit alors de prélever les gaz à travers la capsule en latex, à l'aide d'une seringue graduée, munie d'un robinet à deux voies placé entre le corps de la seringue et l'aiguille. Le piston (4) est ensuite placé en position B jusqu'au prélèvement suivant, évitant ainsi le passage de contenu de rumen dans les conduits (5) et (7). Il est possible d'injecter directement la quantité désirée de gaz à analyser à l'aide de la seringue de prélèvement dans le chromatographe en phase gazeuse.

Technique de prélèvement par ponction. - Les gaz du rumen ont été également prélevés par ponction dans le sac dorsal du rumen à l'aide d'une seringue identique à la précédente, mais dont l'aiguille est remplacée par un trocart « Intranule Vygon * 》 de $2,3 \mathrm{~mm}$ de diamètre ef long de $120 \mathrm{~mm}$.

Analyse des gaz. - Les gaz prélevés ont tous été analysés par chromatographie en phase gazeuse selon une méthode dérivée de celle de Manka (1964) et décrite par Bouvier (1977) puis Jouany ef Senaud (1978).

\section{Validité de la technique de prélèvement proposée (łabl. 1).}

La technique que nous proposons présente trois avantages principaux :

1. - Elle permet d'obtenir un échantillon gazeux totalement dépourvu d'oxygène au cours des heures qui suivent le début de l'ingestion des aliments (tabl. 1). La présence d'oxygène (environ 5 p. $100 \mathrm{du}$ volume totale des gaz) observée $10 \mathrm{~h}$ après le dèbut du repas peut s'expliquer par un ralentissement de l'activité fermentaire entraînant une diminution du pouvoir réducteur du contenu du rumen. L'hypothèse d'une diffusion d'oxygène à partir du sang ne peut être retenue en raison de la faible tension en $\mathrm{O}_{2}$ des tissus. Par contre, les phénomènes successifs de régurgitation et de réingestion des bols alimentaires au cours de la rumination favorisent l'enrichissement en air (azote et oxygène) du mélange gazeux dans le rumen. Il est également possible que la faible pression de gaz dans le rumen à un temps éloigné du repas, favorise la pénétration d'air par l'intermédiaire de la fistule qui n'est pas obstruée de manière étanche par la canule.

2. - Elle est simple à mettre en œuvre et répétable. Nous constatons que les coefficients de variation pour chaque valeur des différents constituants, obtenus selon la méthode proposée, sont faibles (environ 3 p. 100 pour le gaz carbonique); ils sont beaucoup plus importants avec la technique par ponction intraruminale (supérieurs à 8 p. 100) (tabl. 1).

3. - Elle peut être utilisée dans le cadre d'une étude cinétique portant sur l'évolution de la composition des gaz du rumen au cours de la journée puisqu'elle permel de prélever aussi souvent que cela est nécessaire sans perturber l'animal.

* Vygon, BP 7, 95440 Ecouen. 
TABLEAU 1

Analyse de la composition des gaz du rumen selon le mode de prélèvement utilisé (p. 100).

\begin{tabular}{|c|c|c|c|c|c|c|}
\hline & $\begin{array}{l}\text { Temps (heures) } \\
\text { après le repas }\end{array}$ & Hydrogène & Azote & Oxygène & Méthane & Gaz carbonique \\
\hline 1 & $\begin{array}{l}\text { (a) } \\
\text { (b) }\end{array} \ldots \ldots \ldots \ldots \ldots$ & $\begin{array}{l}1,32 \pm 0,06 \\
1,68 \pm 1,35\end{array}$ & $\begin{array}{l}2,99 \pm 0,64 \\
3,06 \pm 2,97\end{array}$ & $\begin{array}{l}\text { non décelable } \\
1,74 \pm 1,62\end{array}$ & $\begin{array}{l}22,83 \pm 0,63 \\
32,03 \pm 4,69\end{array}$ & $\begin{array}{l}72,61 \pm 1,15 \\
62,61 \pm 5,77\end{array}$ \\
\hline 2 & $\begin{array}{l}\text { (a) } \ldots \ldots \ldots \ldots \\
\text { (b) } \ldots \ldots \ldots \ldots\end{array}$ & $\begin{array}{l}1,46 \pm 0,04 \\
1,31 \pm 1,27\end{array}$ & $\begin{array}{l}3,54 \pm 0,53 \\
4,39 \pm 3,00\end{array}$ & $\begin{array}{c}\text { non décelable } \\
1,21 \pm 1,17\end{array}$ & $\begin{array}{l}27,10 \pm 0,84 \\
32,07 \pm 6,09\end{array}$ & $\begin{array}{l}67,96 \pm 1,03 \\
60,96 \pm 5,35\end{array}$ \\
\hline 5 & $\begin{array}{l}\text { (a) } \ldots \ldots \ldots \ldots \\
\text { (b) } \ldots \ldots \ldots \ldots \ldots\end{array}$ & $\begin{array}{l}1,27 \pm 0,05 \\
1,10 \pm 0,97\end{array}$ & $\begin{array}{r}8,61 \pm 0,62 \\
10,39 \pm 2,61 \\
\end{array}$ & $\begin{array}{c}\text { non décelable } \\
1,97 \pm 1,15\end{array}$ & $\begin{array}{l}27,52 \pm 1,01 \\
32,80 \pm 5,70\end{array}$ & $\begin{array}{l}62,60 \pm 2,12 \\
57,32 \pm 4,69\end{array}$ \\
\hline 10 & $\begin{array}{r}(a) \\
\text { (b) }\end{array}$ & $\begin{array}{l}0,97 \pm 0,07 \\
0,05 \pm 0,06\end{array}$ & $\begin{array}{l}10,61 \pm 0,67 \\
13,51 \pm 2,99\end{array}$ & $\begin{array}{l}4,51 \pm 0,64 \\
5,76 \pm 1,86\end{array}$ & $\begin{array}{l}30,51 \pm 1,56 \\
35,72 \pm 8,71\end{array}$ & $\begin{array}{l}53,40 \pm 2,52 \\
44,96 \pm 6,77\end{array}$ \\
\hline 24 & $\begin{array}{l}\text { (a) } \\
\text { (b) } \\
\text { (b) }\end{array}$ & $\begin{array}{l}0,18 \pm 0,06 \\
0,07 \pm 0,06\end{array}$ & $\begin{array}{l}12,71 \pm 0,92 \\
14,63 \pm 3,51\end{array}$ & $\begin{array}{l}4,84 \pm 0,85 \\
4,97 \pm 1,61\end{array}$ & $\begin{array}{l}39,11 \pm 0,96 \\
41,12 \pm 2,56\end{array}$ & $\begin{array}{l}42,34 \pm 0,97 \\
39,60 \pm 3,41\end{array}$ \\
\hline
\end{tabular}

(a) Prélevés selon la technique proposée ; (b) Prélevés par ponction dans le sac dorsal.

Les éfudes de digestion des aliments dans le rumen nécessitent la présence d'une fistule permanente au niveau de cet organe. Notre système permet donc de prélever aisément soit du liquide dans le rumen pour analyser les principaux produits terminaux solubles (acides gras volatils, ammoniaque, acide lactique, etc...) soit les gaz du rumen. Dans la conception actuelle de la canule, il est malgré tout recommandé de ne pas prélever le contenu liquide et les gaz du rumen le même jour afin d'éviter l'entrée d'air au moment de l'ouverture de la canule. Cependant il est possible d'envisager la simultanéité des prélèvements liquides et gazeux. Une sonde placée au fond du sac ventral peut être reliée au bouchon de la canule de manière à prélever du liquide sans entraîner la pénétration d'air à l'intérieur du rumen.

Reçu en novembire 1978.

Accepté en janvier 1979.

\section{Références}

BOUVIER J. C., 1977. Etude descriptive des cinétiques des échanges gazeux du mouton. Influence de la nature de la ration, du niveau d'alimentation et des phénomènes digestifs. Thèse Univ. Clermont II, No d'ordre 81, 124 pp.

JOUANY J. P., SENAUD J., 1978. Utilisation du monensin dans la ration des ruminants. II. Effets sur les fermentations et la population microbienne du rumen. Ann. Zootech., 27, 61-74.

KNOX K. L., BLACK A. L., KLEIBER M., 1969. Sources of rumen carbon dioxide and methane in the laclating dairy cow. J. Dairy Sci., 52, 484-488.

MANKA D. P., 1964. Complete gas chromatographic analysis of fixed bases with one detector using argon as gas carrier. Analyt. Chem., 36, 480-482.

MILTIMORE J. E., MCARTHUR J. M., 1962. Relationship between « in vivo » rumen gas composition and feed efficiency. Nafure, 196, 288-289.

PILGRIM A. F., 1948. The production of methane and hydrogen by the sheep. Austr. J. Sci. Res., Ser. B, 1, 130-138. 